Medicine in the elderly

\title{
Prescribing in the elderly
}

\author{
T Walley, AK Scott
}

A working party of the Royal College of Physicians (RCP) published a report on medication for the elderly over ten years ago, identifying problems and recommending improvements. ${ }^{1}$ Recently, another RCP working party has revisited this subject, and will report later this year. It is therefore appropriate to review the problems of drug use in the elderly, and consider how these have changed in the past ten years.

\section{Demographic changes}

The population in the UK over the age of 70 will increase from 4.76 million in 1985 to 4.93 million by 1995 and then stabilise for 20 years, while those aged over 80 will increase from 0.61 million in 1985 to 0.92 by 1995 and to 1.07 million by 2005. ${ }^{2}$ This will have major consequences for the National Health Service, although less than in some other countries. ${ }^{3}$

\section{Rates of prescribing in the elderly}

Cartwright and $\mathrm{Smith}^{4}$ interviewed 805 elderly people, sampled so as to be representative of national demography, concerning their drug therapy (box 1). Seventy per cent were currently prescribed an average of 2.8 drugs, and $60 \%$ had taken medication within the previous 24 hours. Fifty-nine per cent of drugs had been prescribed for more than two years, $32 \%$ for more than five years, and $16 \%$ for more than 10 years. The majority $(88 \%)$ were issued by repeat prescription and $40 \%$ of patients had not discussed their treatment with a doctor in the previous six months.

Patients' comprehension of their drug therapy was good, with only $9 \%$ having poor or inaccurate knowledge of why a drug had been prescribed. Compliance with drug treatment was claimed by patients to be high $(75 \%)$. There were problems of labelling, including legibility: $20 \%$ understood their doctor to have instructed them to take the drug other than as described on the label. This problem was also identified by the RCP, and the legibility at least should have improved with mandatory printing of labels.

An attempt was made to assess the appropriateness of the drugs, although this is very difficult without detailed knowledge of the patients and their medical history. This found evidence of inappropriate prescribing: $36 \%$ of hypnotics prescribed at doses exceeding the manufacturers' recommendations; duplication of drugs in $4 \%$, (eg, two non-steroidal anti-inflammatory drugs); and potentially harmful interactions in $17 \%$. One worrying finding was that $28 \%$ of drugs reported as prescribed by general practitioners were not known to the patients, while $36 \%$ of drugs reported by patients were not known to the general practitioner. This arises from inaccurate records, inadequate review, perhaps patient hoarding of previous drugs, and probably also from more than one doctor prescribing for the patient.

Purves and Kennedy ${ }^{5}$ collected several months' data on items prescribed from computers in seven general practices (total population 61000 with 10000 over the age of 65). The number of prescriptions, or prescription items, is not a true indicator of volume, since an item could be 10 paracetamol or a three-month supply of an angiotensin-converting enzyme (ACE) inhibitor. A better measure of volume would be the defined daily dose, but data of this kind are not currently available in the UK. Common practice in the UK is that for chronic illness and repeat prescribing, and hence particularly in the elderly, the 'item' is 28 or 30 days supply of a drug. Numbers of prescriptions issued according to age and numbers of concurrent prescriptions are shown in the table. The rates and costs of prescribing increase up to age 74 and then level off, either because patients who reach that age are inherently healthy with less need for drugs, or because a patient can only cope with a small number of drugs. Women were prescribed more than men. Most prescriptions $(80 \%)$ for the elderly were issued as repeat prescriptions, without direct contact between doctor and patient. There were substantial

Therapeutics, University of Liverp PO Box 147, Liverpool L69 3BX, UK T Walley

Department of Geriatric Medicine, University of Manchester, Hope Hospital, Salford M6 8HD, UK AK Scott 
Table Rates of prescribing by age in general practice ${ }^{5}$

\begin{tabular}{lllll}
\hline $\begin{array}{l}\text { Age range } \\
\text { (years) }\end{array}$ & $\begin{array}{l}\text { No of items } \\
\text { per annum }\end{array}$ & $\begin{array}{l}\text { Cost per } \\
\text { annum (£) }\end{array}$ & $\begin{array}{l}{ }^{o} \text { o issued as } \\
\text { repeat } \\
\text { prescriptions }\end{array}$ & $\begin{array}{l}\text { No of concurrent } \\
\text { drugs }\end{array}$ \\
\hline $15-54$ & $4.5-9$ & $27-72$ & $33-36$ & $0.7-1.7$ \\
$65-74$ & 21 & 136 & 80 & 3.5 \\
$75-84$ & 25 & 138 & 80 & 4 \\
\hline
\end{tabular}

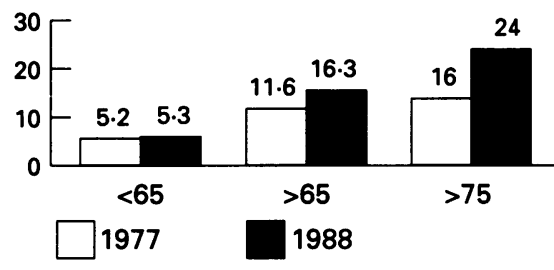

Figure Increases in total prescribing, and prescribing in the elderly and very elderly between 1977 and $1988 .{ }^{2}$ Figures are number of prescribed items/year.
Reasons for the increase in the rate of prescribing for the elderly include:

- reduction of the therapeutic nihilism of doctors in relation to the elderly, eg, in hypertension ${ }^{7,8}$

- increase in the number of very elderly patients, and therefore more morbidity; therapeutic advances for diseases particularly common in the elderly, eg, heart failure

- increased contact between doctors and patients, and increased detection of illness due to the new contract introduced in 1990, which requires doctors to offer regular health checks to many elderly patients

- consumerism, with prescribing in response to perceived patient demand, and a decrease in the stoicism of the elderly population - defensive behaviour by doctors variations between practices: eg, the numbers of concurrent drugs ranged from 2.7 to 4.8 in 65-74-year-old patients, and from 3.2 to 6.7 in those aged $75-84$. This is higher than found by Cartwright and Smith, ${ }^{4}$ and reflects the difference in the number of prescriptions for this age group between 1984 and 1993.

Data are collected on all NHS prescriptions dispensed (as opposed to prescriptions issued ${ }^{5}$ ), including the number of exemptions from prescription charge, and the reason for exemption. Since patients over 65 do not pay prescription charges, prescribing in age-exempt patients in the UK can be tracked (figure). Elderly patients account for about $35-40 \%$ of all items prescribed. The number of items dispensed increased by $17 \%$ overall between 1977 and 1988 , but by $52 \%$ in those over 65 , compared with $1 \%$ in those under $65 .^{2}$

Those over the age of 75 may be particularly heavy drug users, but the exemption data provide no clue to this. Griffin and $\mathrm{Chew}^{2}$ extrapolated from the General Household Survey, which found that those over 75 had $47 \%$ more general practitioner consultations than those aged 65-75, and suggested that the number of prescription items in the very elderly might be similarly increased. They concluded that the number of prescriptions had risen in those aged 65-74 from 11.6 per year in 1977 to 16.3 in 1988, and in the very elderly $(>75)$ from 17 to 24 . This concept is flawed since prescribing rates do not correlate well with consultation rates, especially in the elderly where the majority of prescribing is for chronic illness and covered by repeat prescribing rather than by face-to-face contact. ${ }^{6}$ Nevertheless, the conclusions are broadly supported by other more direct studies, such as that of Purves and Kennedy. ${ }^{5}$

Reasons why the number of prescriptions per elderly patients has increased so much are considered in box 2 .

\section{Results of prescribing: adverse drug reactions}

Elderly patients are more prone to adverse drug reactions for several reasons, including polypharmacy, deterioration in homeostatic mechanisms, and altered pharmacokinetics and pharmacodynamics. Adverse drug reactions are a common cause of admission to hospital. A survey of 2000 admissions to geriatric wards suggested that up to $10 \%$ were due to adverse reactions. ${ }^{9}$ A more recent study $^{10}$ suggests a lower figure of $6.3 \%$, but an important observation in this study was the extent of inappropriate drug therapy, ie, the use of contraindicated or interacting drugs, or unnecessary drugs (as assessed by a hospital doctor) which was responsible for half of the admissions. Other studies from the same group had previously highlighted inappropriate prescribing in residential homes $^{11}$ and the prescription of interacting or contraindicated drugs both by hospital doctors and general practitioners. ${ }^{12}$

Adverse reactions which cause hospital admission are only the more severe end of the spectrum. Of patients living in the community, about $40 \%$ aged over 60 have experienced an adverse drug reaction, compared to $26 \%$ of patients under $60 .{ }^{13}$ Cartwright and $\mathrm{Smith}^{4}$ reported a rate of $15 \%$ : the differences may lie in definitions of adverse drug reaction, and the lower figure is probably an underestimate. American studies, using strict definitions which would not be suitable in the UK, identified inappropriate drug therapy in $25 \%$ of all elderly people living in the community. ${ }^{14}$

Many adverse reactions go undiagnosed. Like many diseases in the elderly, they may present in a nonspecific way, eg, mental confusion, constipation, or unsteadiness. It is important to consider adverse drug reactions more readily in the elderly, if faced with a deterioration in general condition.

These data suggest that there is substantial room for improvement in prescribing for the elderly. Ways ahead might include computer-based decision support about choice of drug or dose, but a more fundamental change in the attitudes and education of doctors and patients about the prescribing of drugs for older patients is necessary also. 


\section{Reasons for excessive and inappropriate prescribing in the elderly include:}

- therapeutic enthusiasm, a doctor's desire to respond to symptoms and problems with treatment, most often drugs

- overenergetic treatment which may lead to incremental prescribing (eg, swollen ankles due to the use of nifedipine for hypertension leads to the use of a diuretic, leading in turn to potassium supplements, dyspepsia, etc)

- patient's (or relative's) demand, real or perceived

- inappropriate response to nonmedical problems

- unrealistic expectations on the part of the doctor, patient or both

- prescribing by rule and failure to individualise treatment for older patients, including a failure to stand back and consider the overall needs of the patient

- inadequate review, leading to failure to discontinue drugs

Box 3

\section{Reasons for inappropriate prescribing}

The reasons for excessive and inappropriate prescribing in the elderly were well defined by the RCP report, ${ }^{1}$ and there is clearly an overlap with the reasons given for increased rates of prescribing in the elderly (box 3 ).

One reason emphasised by the RCP was the failure to make an adequate diagnosis before initiating prescribing. This may result in mistaking manifestations of aging for disease, eg, prescribing prochlorperazine for postural unsteadiness, or diuretics for gravitational oedema. It must be recognised that 'inadequate clinical assessment' before prescribing is often inevitable. The vast majority of prescribing is undertaken by general practitioners, who are seeing patients often under difficult circumstances, with limited access to the type of diagnostic investigation commonplace in hospital, and with many factors related to the patient other than their immediate medical condition in mind. Diagnoses are often not clear when a patient presents at an early stage of illness. The general practitioner must therefore make a best formulation of a patient's problems, rather than a definitive diagnosis, and accept a high degree of uncertainty. Inevitably, given the nature of the consultation in primary care, some prescribing will seem, especially in hindsight, to have been pharmacologically inappropriate. ${ }^{15}$ Hospital doctors should temper their criticism of general practitioners in this regard by better understanding.

Another influence on prescribing is pharmaceutical industry promotion, which encourages prescribing in response to all problems, and the use of inappropriate drugs for the elderly, eg, ACE inhibitors for hypertension. While it is a company's legitimate right to promote a drug in accordance with its licence, this does not always meet the needs of rational prescribing, and companies might consider their duties in this.

\section{Prescribing at the interface between hospital and community}

Many hospital geriatricians attribute their clinical reputation to stopping drugs started by other doctors, but it is difficult to achieve the ideal of moderation in medication, even in hospital. One study showed that the number of drugs per patient did not change between admission and discharge. ${ }^{16}$ There is clearly cause for concern about prescribing for elderly patients within hospital, where, despite support, problems may arise. ${ }^{12}$

A particular concern is what happens to patients on discharge. In Abram's study, ${ }^{16}$ an eighth of the prescribed discharge drugs were stopped within three weeks, mainly by patients themselves, and replaced by an equal number of drugs, half of which were done by patients without medical advice. In a more recent study, the drug therapy of 45 out of 50 patients was altered within two weeks of discharge from a geriatric ward. ${ }^{17}$ These changes included switching of branded for generic products and minor alterations of timing or instructions; these may confuse patients and contribute to poor compliance. Eleven patients changed dose, 10 stopped drugs and 20 had new drugs added. Many of these changes were initiated by patients without medical advice. Other problems included restarting previous or hoarded drugs, and inadequate drug history on admission (particularly over-the-counter drugs). There was a serious lack of continuity of care in prescribing between hospital and primary care. Ways to improve this have included better communication between professionals, more instruction (especially written) for patients and self medication by patients holding their own drugs for a few days prior to discharge: the latter is particularly popular among nursing staff, and a recent evaluation suggests this may be useful. ${ }^{31}$

Reviewing medication is essential in improving prescribing in the elderly. The general practitioner may be uncertain of the continuing need for drugs initiated by hospital doctors, when a patient is no longer under review by the hospital, and may be reluctant to stop such a drug. 'Shared' responsibility sometimes results in no responsibility. One persons needs to be clearly accountable for a patient and this should be the general practitioner. Hospital doctors should indicate clearly the indication for drug therapy, its monitoring and likely duration of therapy; this happens all too rarely.

\section{Clinical pharmacology in the elderly}

There are many excellent reviews of how aging alters responses to drugs and how the body handles drugs ${ }^{18,19}$ (box 5). The clinical importance of many of these changes has tended to be overstated. The most important and predictable change is a reduction in renal drug clearance, in line with age-related changes in renal function. This is a problem for drugs which are mainly excreted unchanged by the kidney and which have a narrow therapeutic range (eg, digoxin, amino-

\section{Comment}

This is a hypothetical case, but most doctors can tell of similar cases they prescribing arises from a knee jerk reaction to individual problems arising from adverse drug reactions, without adequate clinical assessment or taking a wider view of the patient's needs 
Clinical pharmacology in the elderly: differences from younger patients

Major pharmacokinetic differences:

- reduced first pass metabolism

- reduced renal drug clearance

Major pharmacodynamic differences:

- impaired homeostatic mechanisms

- increased susceptibility to many drug effects

Box 5 glycosides, or lithium); the doses of these may need to be modified. First-pass metabolism also tends to be reduced with a resulting increase in bioavailability for those drugs which undergo extensive first-pass metabolism (eg, propranolol, verapamil, metoclopramide, many opioids). Hepatic clearance of drugs from the systemic circulation decreases slightly with age; this is not a problem in most elderly patients, but may be in the very frail elderly, where the decrease may be more dramatic. All drugs should be used with particular caution and in very low doses in such patients. Changes in drug absorption or protein binding are rarely of clinical importance in the elderly.

Responses to drugs may be altered for reasons other than a change in drug concentration. For instance, the elderly are more susceptible to benzodiazepines than younger patients. The normal homeostatic mechanisms may be impaired, so that postural hypotension becomes a more common response to antihypertensives than in younger patients. Warfarin requirements are usually about $25 \%$ less than in younger patients.

In the past, new drugs were often poorly studied in the elderly before drug launch. This led to many problems, of which the best known is benoxaprofen (Opren), a non-steroidal anti-inflammatory drug which caused serious skin and hepatic reactions in the elderly. Subsequent studies showed that the half-life of this drug in the elderly was greatly increased, leading to drug accumulation, and probably many of the adverse effects. Data on the effects of a drug in elderly subjects must now be submitted before it is licensed for use in the elderly. Nevertheless, it is a fundamental principle that new drugs should be prescribed with great caution, especially in patients at high risk of adverse effects such as the elderly; this is often forgotten.

\section{Compliance}

Adherence to long-term medication is often poor. ${ }^{20}$ This is difficult to study and may not be considered a source of therapeutic failure. Up to $75 \%$ of elderly patients may not adhere to prescribed medication, with clinically significant results, such as disease breakthrough, in $25 \%{ }^{1}$ Patients may hoard unused drugs for future use, leading to more confusion for both patient and doctor. Reasons for noncompliance include poor instruction, excessively complex drug regimens, differing perceptions of what is important by doctor and patient, adverse drug reactions, and inconvenience. While doctors may consider noncompliance as a form of deviant behaviour, for many elderly patients there may be degrees of intelligent noncompliance and self preservation. Underlying all of these is a failure of dialogue between doctor and patient, so that each has failed to explain to the other what is happening and what they wish to happen. The community pharmacist can be a less hurried and more approachable source of information for the patient. Doctors can have difficulty in identifying noncompliers: there may be clues in a patient's poor attendance at surgery, or inconsistent requests for new supplies of medication. The risk of noncompliance should be reduced as much as possible by simplifying drug regimens, avoiding polypharmacy, and careful instruction, preferably in writing, to inform and motivate the patient. Once-a-day dosage forms may be useful in individual patients, although in general their advantages are overplayed. Pharmacists can assist with the use of such devices as reminder boxes to dispense a week's supply of medication. None of these are complete answers: doctors need to bear the likelihood of noncompliance in mind, particularly in assessing response to therapy, and to reinforce the instruction at every opportunity (box 6).

Face-to-face review encourages proper use of therapy; without such review, there is underuse of most drugs, but overuse in the case of sedatives. ${ }^{5}$ Repeat prescribing, the most common means of prescribing in the elderly, is sometimes used as a mechanism by both patients and doctors to avoid contact. Careful repeat prescribing systems, including review at predefined intervals, are now actively encouraged by health authorities in primary care. Such systems can be manual or computer based. Advantages of computer-based systems include speed, accuracy, legibility and convenience. More sophisticated systems may also offer decision support on appropriate doses for the elderly, interactions, contraindications and previous adverse reactions, and avoid some of the grossest errors in precribing. Computers are less likely to address the vital question of whether to prescribe at all.

One other trend in prescribing in recent years may adversely affect compliance: the rate of prescribing by generic rather than brand name has risen over the past few years, encouraged by government. Elderly patients may thus receive a given drug in tablets of differing appearance which may confuse. This can be avoided by patient education and cooperation between doctor and pharmacy. consider the possibility of poor compliance

clear dialogue between patient and doctor

- regular face-to-face review of medication by doctor and patient

written drug regimens 


\section{Education of prescribers}

Prescribing for the elderly is considered by academic clinical pharmacologists to be the single most important item that they can teach medical students, ${ }^{21}$ and all medical schools claim to do this. However, there are still deficiencies which need to be addressed by postgraduate education. Therapeutics is now a popular subject in postgraduate centres, in contrast to 10 years ago, but the agenda for such education is often set by a pharmaceutical company, and is not likely to be a geriatrician or clinical pharmacologist encouraging less prescribing, rather than more. Medical Audit Advisory Groups are also tackling prescribing in the elderly, and need every encouragement and support.

\section{Nursing and residential homes}

Certain groups of the elderly are at particular risk of overprescribing, including those living in residential and nursing homes. The volume and costs of prescribing in such patients are staggering - perhaps four times as much for nursing home patients as for patients living in the community, ${ }^{22}$ and not all accounted for by differences in morbidity between the two groups; $86 \%$ of patients in long-term residential homes are on long-term medication, receiving a median of three drugs per day. ${ }^{23} \mathrm{~A}$ small review in one nursing home suggested that the original indication had become completely irrelevant for $19 \%$ of drugs, while the role of a further $56 \%$ of drugs was considered uncertain ${ }^{24}$; a larger review within one practice suggested that for elderly patients living in the community, the comparative figures were $10 \%$ and $28 \% .{ }^{25}$

A particular area for concern is the overuse of benzodiazepines $-23 \%$ of patients in residential homes were receiving hypnotics on a long-term basis in one study, often the long-acting nitrazepam..$^{23}$ The rate of use of hypnotics varied between homes from $3.6 \%$ to $60 \%$, which surely says more about the philosophy of care in the homes than about the patients. Nonprescribed drugs may also be widely used in such homes. ${ }^{27}$ There are concerns about untrained staff dispensing drugs in residential homes, which leads to problems such as the use of drugs for patients other than those for whom the drug was prescribed, and administration errors including over or under dosing. ${ }^{26,27}$

In the US, by law, all nursing homes (in general, rather larger than those in the UK) must employ a pharmacist to review prescribed medication every month, and advise on drug choice and duration of therapy. ${ }^{28}$ Cost containment and education of staff is also part of their remit. The final decision for the prescription continues to rest with the doctor, but he is required by law to consider the advice tendered by the consultant pharmacist (the advice is accepted in $70 \%$ of cases).

Such a scale of pharmacist involvement in the UK is less likely. At present pharmacists are paid a fee to visit residential homes and advise on drug storage and administration. ${ }^{29}$ In only $6 \%$ of such visits is the quality of prescribing, or staff education considered. Work in the US has shown staff education to reduce the use of psychotropic medication in nursing homes. ${ }^{30}$ Many doctors and staff in residential or nursing homes would welcome assistance in prescribing. Health authorities and government might usefully provide funding to support such initiatives.

\section{Conclusions}

What are the possible solutions to the problems with current prescribing in the elderly? A start would be the application of basic rules of thumb, such as assess the patient clinically as best one can; review patients and drugs regularly; simplify drug regimens; encourage good compliance and communicate well with the patient; familiarise one's self with a narrow range of drugs; use low doses, and increase doses slowly; consider the patient as a whole and avoid overtreating when a patient's physical or mental problems suggest otherwise. Most important of all, if in doubt, don't prescribe at all. These are good general rules for prescribing, but apply particularly to a vulnerable group like the elderly. Better education for all concerned, patients and doctors, would be helpful. The use of computers and other means of decision support would fine tune these suggestions. The recommendations of the RCP working party can be awaited with interest.

\section{Box 7}

3 Laing W, Hall $M$. The challenges of aging: agenda for health. London: Association of the British Pharmaceutical Industry, 1991.

4 Cartwright A, Smith C. Elderly people: their medicines and their doctors. London: Routledge, 1988.
1 Royal College of Physicians. Medication for the elderly. $\mathcal{F} R$ Coll Physicians Lond 1984; 18: 7-17.

2 Griffin JP, Chew R. Trends in usage of prescription medicines by the elderly and very elderly between 1977 and 1988. London: Association of the British Pharmaceutical Industry, 1990.

appropriate prescribing in the assessment

needs to be balanced by a consideration

- avoid polypharmacy

regularly, and discontinue unnecessary drugs

- use low doses and increase slowly
5 Purves I, Kennedy J. The quality of general practice repeat prescribing. University of Newcastle upon Tyne: Dept of Primary Health Care, 1994. 
6 Cartwright A. Medicine taking by people aged 65 or more. In: Denham M, George CJ, eds. Drugs in old age: new perspectives. Edinburgh: Churchill Livingstone, 1990.

7 MRC Working party. MRC trial of treatment of hypertension in older adults. $B M \mathcal{F} 1992$ 304: 405-12.

8 Fahey T, Silagy C. General practitioners' knowledge of and attitude to the management of hypertension in elderly patients. $\mathrm{Br} F \mathrm{Gen}$ Pract 1994; 44: 446-9.

9 Williamson J, Chopin JM. Adverse drug reactions to prescribed drugs in the elderly; a multicentre investigation. Age Ageing 1980; 9: multice.

10 Lindley CM, Tully MP, Paramsothy V, Tallis $R$. Inappropriate medication is a major cause of adverse drug reactions in elderly patients. Age Ageing 1992; 21: 294-300.

11 Gosney M, Valet C, Tallis R, Oddment E, A-Hames S. Inappropriate prescribing in Part 3 residential homes for the elderly. Health Trends 1989; 21: 129-31.

12. Gosney M, Tallies $R$. Prescription of contraindicated and interacting drugs in elderly patients admitted to hospital. Lancet 1984; ii: 564-7.

13 Denham M. Adverse drug reactions. In: Denham M, George CJ, eds. Drugs in old age: new perspectives. Edinburgh: Churchill Livingperspectives.

14 Wilcox S, Himmelstein D, Woolhandler S. Inappropriate drug prescribing for the community dwelling elderly. $\mathscr{f} A M A$ 1994; 272 $292-6$.
15 Reilly P, Marinker M. Rational prescribing: can it be judged? In: Marinker M, ed. Cont roversies in health care policies. London: $\mathrm{BM}$ J Publishing Group, 1994.

16 Abrams J, Andrews K. The influence of hospital admission on longterm medication of elderly patients. $¥ R$ Coll Physicians (Lond) 1984; 18: 225-7.

17 Cochrane RA, Mandal AR, Leger Scott M, Walker $R$. Changes in drug treatment after discharge from hospital in geriatric patients. discharge from hospital in

18 Dawling S, Crome P. Clinical pharmacokinetic considerations in the elderly: an macokinetic considerations in the elderly: an update. Clin

19 Anon. Prescribing for the elderly. British National Formulary. London: British Medica Association and Royal Pharmaceutical Society, $1994 ; 28$

20 Wright EC. Noncompliance - or how many aunts has Matilda? Lancet 1993; 342: 909-13.

21 Walley $T$, Bligh J, Orme $M$, Breckenridge AM. Undergraduate teaching in clinical pharmacology and therapeutics: responses to future developments. $\mathrm{Br} \mathcal{F}$ Clin Pharmacol 1994; 37: 137-43.

22 Crompton B. GPs need more PUs for nursing home patients. Prescriber 1995; 6: 44-5.

23 Weedle PB, Poston JW, Parish PA. Use of hypnotic preparations by elderly people in residential homes. $\exists R$ Coll Gen Pract 1988; residential

24 Bruce S. Regular prescribing in a residential home for elderly women. $B M F$ 1982; 284: 1235-7.
25 Tulloch AJ. Repeat prescribing for elderly patients. BMF 1981; 282: 1672-5.

26 Weedle P, Poston W, Parish PA. Survey of remedies held and dispensed in residential homes for the elderly. $\mathcal{F} R$ Coll Gen Pract 1987; 37: 412-3.

27 Gilligan JD. Prescribing in general practice. Occasional paper 54. London: Royal College of General Practitioners, 1991.

28 American Society of Consultant Pharmacists (ASCP). Long term care pharmacy; the new market. Arlington: ASCP, 1989.

29 Pharmaceutical Society. Administration and control of medicines in residential homes. control of medicines in residentir

30 Avorn J, Soumerai S, Everitt D, et al. A randomised program to reduce the use of psychoactive drugs in nursing homes. $N$ Engl f Med 1992; 327: 168-73.

31 Lowe C, Raynor D, Courtney E, Purvis J, Teale C. Effect of self-medication programme on knowledge of drugs and compliance with treatment in elderly patients. $B M \mathcal{F} 1995 ; 310$ : $1229-31$.

James Bryam Herrick (1861-1954) was born in Oak Park, Illinois, which his maternal grandmother had reached by covered wagon, after emigrating from England to the US. He graduated MD (1888) at Chicago's Rush Medical College. He was the first to describe "peculiar elongated sickle-shaped red blood corpuscles in a case of severe anaemia" (1910), later to be termed sickle cell anaemia. He was also a pioneer in describing coronary occlusion and angina pectoris (1912). 